\title{
Integrated analysis of aerodynamic/stealth characteristics of blended-wing-body lifting vehicle
}

\author{
Ye Tao ${ }^{1}$, Jianquan $\mathrm{Ge}^{1}$, Qiangqiang $\mathrm{Xu}^{1}$, Hao Huang ${ }^{1}$ and Tao Yang ${ }^{1, a}$ \\ ${ }^{1}$ College of Aerospace Science and Engineering, National Univ. of Defense Tech., 410073, Changsha, P.R. China
}

\begin{abstract}
In this paper, a blended-wing-body lifting vehicle is designed, with the cross section described by Class/Shape transformation method (CST), the outline controlled by an exponential function, and tail regulated by B-spline curve. Rapid estimation procedures of aerodynamic based on plane method and of radar cross section (RCS) based on physical optics are developed and validated. A frame of integrated design and analysis of aerodynamic and stealth characteristics is constructed and applied to parameter sensitivity analysis. The results show that the parameters that affect the aerodynamic differ from those of RCS, which means the vehicle could be good at both aerodynamic and stealth, and the study followed should be focused on it.
\end{abstract}

\section{Introduction}

The design of hypersonic glide vehicle (HGV) has been a hot field in vehicle research for a long time. It involves disciplines like aerodynamic, thermal, structural, trajectory, guidance, stealth and so on, which have strong coupling effects between each other. The coupling effects should be fully considered in the overall design process. However, the design of HGV is general guided by the aerodynamic characteristics currently, which induced the appearance of many high lift-to-drag ratio shapes[1] in the laboratory and paper, it is doubtful that whether these shapes possess practical significance. Against this problem, some scholars have proposed that introducing new constraints or evaluation criteria like the loading rate[2], structure[3], heat[4], stability[5] and so on. But none of these are related to the shape directly. This paper argues that, in the shape design of HGV, the capability of stealth should be treated as equal as aerodynamic. Not only because the shape feature is the most important technology to improve the stealth performance, but also the low observable technology is the most efficient technique to against the rapid development of supersonic aircraft defense system and improve the penetration ability of assault weapons.

Lots of work has been carried out about the integrated design of aerodynamic and stealth. Chu[6] develops a mixed RCS calculation method to calculate the radar scattering performance of a kind of cruise missile. Liu[7] introduces three control factors to accelerate the multi-layer fast multi-level algorithm and ensure the appropriate accuracy meanwhile. In order to reduce the computational cost, the optimization process is divided into two levels by $\mathrm{Wu}[8]$, the first is the coupling optimization of aerodynamic and stealthy, and the effect of structure is considered later. These are intended to improve

a Corresponding author: Ty93pical@163.com 
the precise and accelerate the computation of RCS. There are also works focus on the structure of integrated modeling[9-12], and the main purpose of them is to acquire an efficient operation system of multi-discipline.

In summary, the aerodynamic and stealth integrated design technology of low speed of fighter planes, unmanned aerial vehicles and cruise missile is pretty mature, but the corresponding research on $\mathrm{HGV}$ is relatively few. In this paper a simple parameterization method for hypersonic Blended-wing-body Lifting Vehicle (BLV) is proposed, and then an integrated platform established with rapid estimation modules of aerodynamic and RCS, an experiment is designed to obtain the sensitivity of shape parameters on the aerodynamic or stealth properties, which lay the foundation for shape optimization study subsequently.

\section{Parameterization for blended-wing-body lifting vehicle}

Geometric modeling is the prerequisite for performance analysis. The shape parameter system is the basis of geometric modeling. A kind of lifting vehicle with blended wing is proposed, which is based on the parameterization of Class/Shape transformation method (CST), exponential function method and B-spline curve method. The aircraft is mainly divided into two parts: the fuselage and body-merged tail. The cross section of fuselage is described with CST method, the outline is controlled by an exponential function, and the tail is regulated by B- spline curve. The vehicle designed is shown in figure 1.

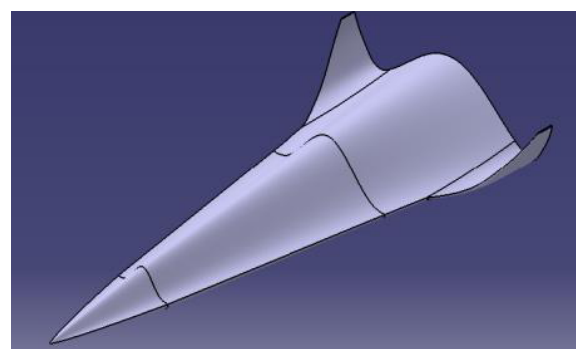

Figure 1. The blended-wing-body lifting vehicle

\subsection{Parameterization}

The Class/Shape Transformation Method is proposed by Kulfan and Bussoletti[13-15], which use the combination of Class Function and Shape Function to express the geometric Shape change. The formula for CST curve is

$$
\eta(\psi)=C(\psi) S(\psi)+T(\psi)
$$

In the formula, $\psi$ is a normalized length, $C(\psi)$ is the Class Function, which represents the geometric property of the curve, $S(\psi)$ is the shape function, which represents the shape of the curve precisely. $T(\psi)$ is the thickness function. In the design of the cross section of the fuselage, the thickness function is assumed as $T(\psi)=0$, and the cross section is divided into the upper and lower surfaces to be controlled by two curves, as shown in figure 2 .

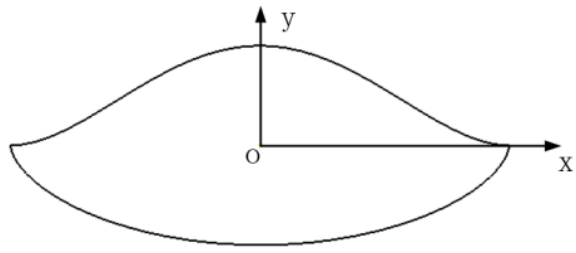

Figure 2. CST cross section curve

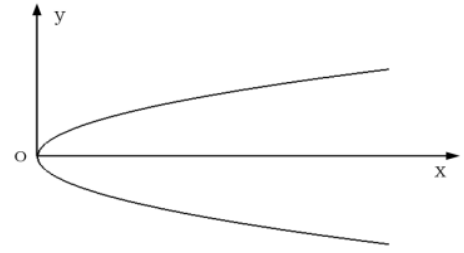

Figure 3. Exponential function control line 
The width of the section is set to $W$, so the range of $x$ is [-W/2, W/2], and the height of the curve is set to $H$, so the range of $y$ is $[0, H]$. With $C(\psi)=\psi^{N c 1}(1-\psi)^{N c 2}$ and $S(\psi)=2^{2 N c}$, the control curve of the upper and lower surface can be expressed as

$$
\begin{aligned}
& y_{u}=H_{u} \cdot(x / W+0.5)^{N c u} \cdot(0.5-x / W)^{N c u} \cdot 2^{2 N c u} \\
& y_{l}=-H_{l} \cdot(x / W+0.5)^{N c l} \cdot(0.5-x / W)^{N c l} \cdot 2^{2 N c l}
\end{aligned}
$$

The subscript $u$ and $l$ are denoted as upper and lower curve parameters. Thus, each section will be controlled by five parameters, which are $H_{u}, H_{l}, N c u, N c l$ and $W$.

The outline of the fuselage is controlled by an exponential function, which is $y=0.5 \mathrm{~W} \cdot(x / L)^{n}$, where $W$ is the maximum width of the model, $L$ is the total length, $n$ is the index to control variation of the curve. An example of the outline generated by exponential function shown in Figure 3.

A B-spline curve is controlled by a series of key point, the more of control points, the finer the curve is. Set $B_{i, p}$ as $P$ order B-spline basis function, $P_{i}$ as the coordinates of the main point, $A(t)$ as a parametric curve. The mathematical expression of $A(t)$ is:

$$
\mathrm{A}(\mathrm{t})=\sum_{i=1}^{n+1} B_{i, p}(t) P_{i}
$$

Figure 4 explains how to use the B-spline curve to control the tail. Point $\mathrm{A}$ is adhered the longitudinal section of the body and fixed, due to the limitation of the overall width, the coordinate value of the direction $X$ of point $\mathrm{D}$ is fixed too. Thus, the main control points are point $\mathrm{B}$ and $\mathrm{C}$. In order to ensure the rationality of the two control points, the heights are fixed too, and only the values of the coordinate $X$ are changeable.

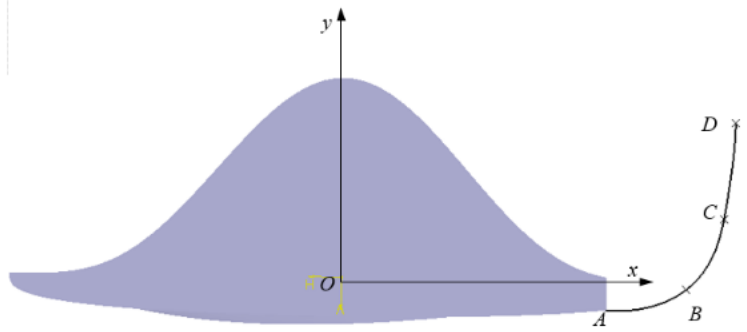

Figure 4. Curve controlled by B-spline method

The tail could be formed by using multi-section solid technique guided by the B-spline mentioned above. The sections used are the longitudinal section in point $\mathrm{A}$ and normal planes in points $\mathrm{B}, \mathrm{C}$ and $\mathrm{D}$, which are generated by narrowing 0.66 times in sequence based on the section in point $\mathrm{A}$.

\subsection{Main parameters of the BLV}

The fuselage is controlled by three sections, and there are six parameters $\left(H_{u i}, H_{l i}, N c u_{i}, N c l_{i}, l_{i}\right.$ and $\left.W_{i}\right)$ for each section, along with $n$ the index of exponential function and overall length $L$, there are 20 parameters to generate the body in summary. Besides, the tail is simplified to be controlled by the coordinates $X$ of point $B\left(a_{I}\right)$ and $C\left(a_{2}\right)$, plus the total width $W$, a total of 23 parameters make up the system of BLV's parameterization. All parameters used to control the shape of BLV are presented in table 1, along with their symbol, initial value and range.

Table 1. Main parameters of the BLV

\begin{tabular}{|l|c|c|c|c|c|c|c|}
\hline No. & Symbol & Initial Value & Range & No. & Symbol & Initial Value & Range \\
\hline $\mathbf{1 .}$ & $L$ & $6500 \mathrm{~mm}$ & - & $\mathbf{1 3 .}$ & $H_{u 2}$ & $650 \mathrm{~mm}$ & $(550,700)$ \\
\hline $\mathbf{2 .}$ & $W$ & $2960 \mathrm{~mm}$ & - & $\mathbf{1 4 .}$ & $H_{l 2}$ & $120 \mathrm{~mm}$ & $(100,250)$ \\
\hline
\end{tabular}




\begin{tabular}{|l|c|c|c|c|c|c|c|}
\hline $\mathbf{3 .}$ & $l_{l}$ & $1500 \mathrm{~mm}$ & - & $\mathbf{1 5 .}$ & $N_{c u 2}$ & 4 & $(1.32,5)$ \\
\hline $\mathbf{4 .}$ & $l_{2}$ & $3000 \mathrm{~mm}$ & - & $\mathbf{1 6 .}$ & $N_{c l 2}$ & 0.3 & $(0.3,0.62)$ \\
\hline $\mathbf{5 .}$ & $l_{3}$ & $2000 \mathrm{~mm}$ & - & $\mathbf{1 7 .}$ & $W_{3}$ & $2500 \mathrm{~mm}$ & - \\
\hline $\mathbf{6 .}$ & $n$ & 0.8 & $(0.5,0.85)$ & $\mathbf{1 8 .}$ & $H_{u 3}$ & $750 \mathrm{~mm}$ & $(700,900)$ \\
\hline $\mathbf{7 .}$ & $W_{l}$ & $800 \mathrm{~mm}$ & $(600,900)$ & $\mathbf{1 9 .}$ & $H_{l 3}$ & $150 \mathrm{~mm}$ & $(150,250)$ \\
\hline $\mathbf{8 .}$ & $H_{u l}$ & $320 \mathrm{~mm}$ & $(250,450)$ & $\mathbf{2 0 .}$ & $N_{c u 3}$ & 4 & $(1.32,5)$ \\
\hline $\mathbf{9 .}$ & $H_{l l}$ & $120 \mathrm{~mm}$ & $(100,250)$ & $\mathbf{2 1 .}$ & $N_{c l 3}$ & 0.3 & $(0.3,0.62)$ \\
\hline $\mathbf{1 0 .}$ & $N_{c u l}$ & 4 & $(1.32,5)$ & $\mathbf{2 2 .}$ & $a_{l}$ & 1400 & $(1350,1410)$ \\
\hline $\mathbf{1 1 .}$ & $N_{c l l}$ & 0.3 & $(0.3,0.62)$ & $\mathbf{2 3 .}$ & $a_{2}$ & 1450 & $(1410,1470)$ \\
\hline $\mathbf{1 2 .}$ & $W_{2}$ & $1800 \mathrm{~mm}$ & $(1650,1800)$ & & & & \\
\hline
\end{tabular}

\section{Method of aerodynamic computation}

In the conceptual design of a new vehicle, a large number of aerodynamic characteristics prediction is needed. Due to the huge expenditure of time, manpower and money of numerical computation, it is necessary to develop a fast approximation method in the initial stage.

It is difficult to calculate the aerodynamic characteristics in hypersonic flows precisely for a complex object. But for a simple facet, things are different, the expenditure of time could be much less and the precise could be relatively high with Plane Method. So it would be a good idea to create a method by dividing complex curved surface into small triangle surface and calculate the pressure coefficient on each face.

The plane method of aerodynamic is based on the modified Newton method, which is aimed at the situation presented in figure 5, the pressure coefficient of plane $\mathrm{A}$ is $C_{p}=C_{p \max } \sin ^{2} \theta$, where $C_{p \max }$ is the maximum pressure coefficient, and in the hypersonic flows, $C_{p \max }$ is calculated on the stationary point of the normal shock, which is:

$$
C_{p \max }=\frac{2}{\gamma M_{1}^{2}}\left\{\left[\frac{(\gamma+1) M_{1}^{2}}{4 \gamma M_{1}^{2}-2(\gamma-1)}\right]^{\frac{\gamma}{\gamma-1}}\left(\frac{1-\gamma+2 \gamma M_{1}^{2}}{\gamma+1}\right)-1\right\}
$$

As shown in figure $6, \mathbf{n}$ is the normal vector and $G$ is the geometric center of a facet, $\mathbf{V}_{\infty}$ is the velocity of the free stream, which would be $\mathbf{V}$ after the impact with the facet, and the included angle between $\mathbf{V}_{\infty}$ and $\mathbf{V}$ is $\Theta$ :

$\Theta=\frac{\pi}{2}-\arccos \left(\frac{\mathbf{V}_{\infty} \cdot \mathbf{n}}{\left|\mathbf{V}_{\infty}\right| \cdot|\mathbf{n}|}\right)$

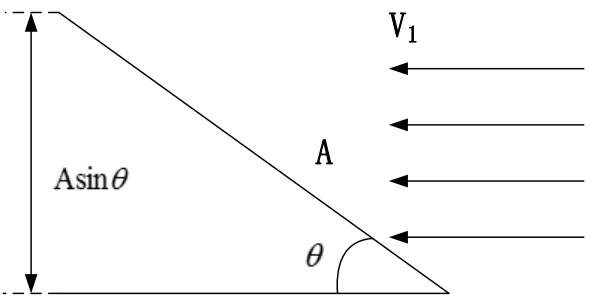

Figure 5. Sketch of the modified Newton method

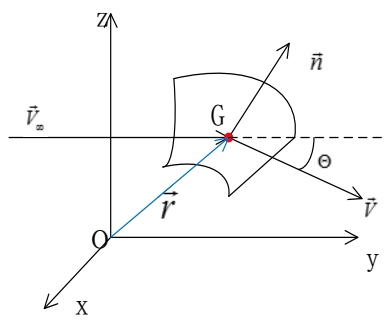

Figure 6. Sketch of the plane method

With $C_{p}, \mathbf{n}, G$ and the area $A$ of every facet, the overall aero force is expressed as followed:

$$
\mathbf{F}=-\int_{s} p \mathbf{n} d s=-\sum_{i=1}^{n_{e}} p_{i} n_{i x} d s_{i} \mathbf{i}-\sum_{i=1}^{n_{e}} p_{i} n_{i y} d s_{i} \mathbf{j}-\sum_{i=1}^{n_{e}} p_{i} n_{i z} d s_{i} \mathbf{k}
$$

where, $p=C_{p} \cdot A$, and $n_{e}$ is the number of facets. 
In order to verify the rapid estimation procedure of the aerodynamic characteristics developed in this paper, eight angles of attack $\left(1^{\circ}, 2.5^{\circ}, 4^{\circ}, 5^{\circ}, 5.5^{\circ}, 6.5^{\circ}, 8^{\circ}, 10^{\circ}\right)$ were calculated for the BLV model with the non-stick CFD program Cart3D in the free stream whose Mach number is 7 , the height is $25 \mathrm{~km}$, the reference area is $1 \mathrm{~m}^{2}$, and the reference length is $6.5 \mathrm{~m}$.

The lift-to-drag ratios of estimation procedure and Cart3D are compared at the angle of attack from 1 to 10 degrees, and plotted in figure 7 for conspicuous. The results shows that the estimation procedure is dependable for that the trends for the two methods are similar and the numerical error between are regular and easily corrected, and the relative error in the maximum lift-to-drag ratio is only $5.2 \%$, which is pretty acceptable for an estimation method.

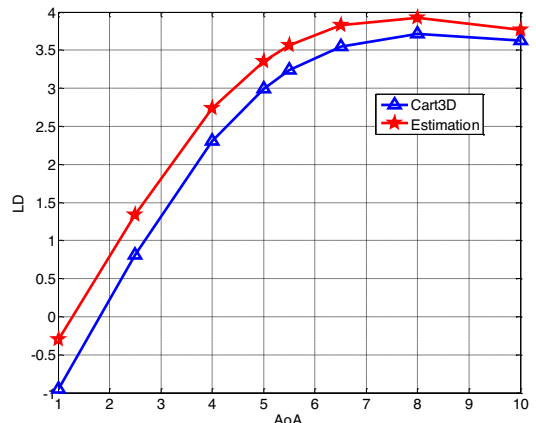

Figure 7. Sketch of aerodynamic compare

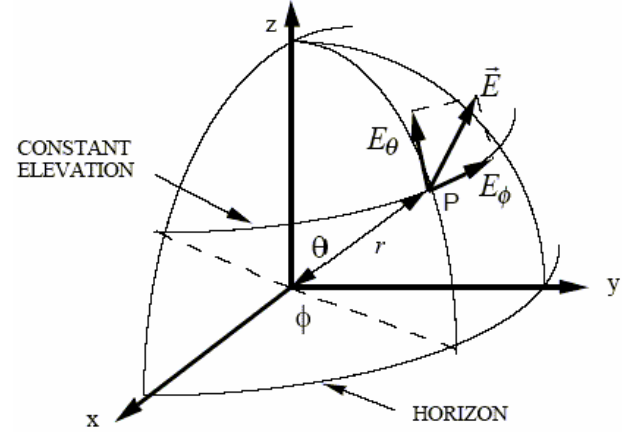

Figure 8. Coordinate system of physical optics

\section{Method of electromagnetic scattering calculation}

\subsection{RCS definitions}

The scattering energy of radar target can be expressed by the product of an effective area and the incident power density, which is called radar cross section $\sigma$, and the basic theoretical definition formula is:

$$
\sigma=\lim _{R \rightarrow \infty} 4 \pi R^{2} \frac{\left|\mathbf{E}_{s}\right|^{2}}{\left|\mathbf{E}_{i}\right|^{2}}=\lim _{R \rightarrow \infty} 4 \pi R^{2} \frac{\left|\mathbf{H}_{s}\right|^{2}}{\left|\mathbf{H}_{i}\right|^{2}}
$$

Where $\mathbf{E}_{s}$ and $\mathbf{H}_{s}$ is the energy intensity of the scattering electric field and magnetic field at the point whose distance to the goal is $\mathrm{R}, \mathbf{E}_{i}$ and $\mathbf{H}_{i}$ is the energy intensity of the incident electric field and magnetic field. Considering that the RCS varies greatly, it is usually expressed in logarithmic form, $\sigma_{d B s m}=10 \lg (\sigma)$, and its unit is dBsm, meaning the decibel of the square meter.

RCS is a function of the target shape, material, electromagnetic wave frequency, polarization type and target attitude. Normally, RCS is designated as a symbol $\sigma_{p q}(\theta, \varphi)$ after the target is determined. Where $p$ and $q$ are the polarization type of the wave, $\theta$ and $\varphi$ is the relative angle to the line of sight[16].

\subsection{Physical optics method}

The physical optics method is for electrically large targets in the high frequency, the source of the scattered field is replaced by the induced current on the surface in the theory, and then the surface induced current is integrated to obtain the scattering field. Assuming that the radius of curvature of the surface of the scattering is much larger than that of the wavelength, it is considered that the influence of the scattering from the other surface of the object can be neglected during the solution, so that the RCS of each surface element can be solved independently.

As shown in Figure 8, suppose there is a radiation source in the origin, the scattering field in $P$ is: 


$$
\mathbf{E}_{s}(r, \theta, \varphi)=E_{\theta}(r, \theta, \varphi) \hat{\theta}+E_{\varphi}(r, \theta, \varphi) \hat{\varphi}=\frac{-j K Z_{0}}{4 \pi r} e^{-j k g} \iiint_{V} \mathbf{J} e^{j k g} d v^{\prime}
$$

where, $r$ is the distance between point $P$ and the origin, $\theta$ and $\varphi$ is the Euler transition angle, $\mathbf{J}$ is the induced current, $Z_{0}$ is the inherent impedance of surrounding space, $k=2 \pi / \lambda$ ( $\lambda$ is the wavelength) is the wave number, $g$ is defined as $g=\mathbf{r} \cdot \mathbf{r}^{\prime}$. When the scattering center is a triangular element, the volume integral can be converted into surface integral, which is

$$
\mathbf{E}_{s}(r, \theta, \varphi)=\frac{-j K Z_{0}}{4 \pi r} e^{-j k g} \iint_{A} \mathbf{J}_{s} e^{j k g} d s_{p}
$$

Where $\mathbf{J}_{s}$ is the surface current, $A$ is the area of the facet. Using the integral formula in all surface element, and the total scattering field of the target could be acquired by summing them up. In order to ensure the accuracy of calculation, the length of grid should generally set to $1 / 10 \sim 1 / 3$ of the wavelength.

\subsection{Results and verification}

The monostatic RCS characteristics of a cylinder and the BLV are calculated and compared with the commercial software CST and the experimental data.

Cylinder: length $71.1 \mathrm{~cm}$, diameter $14 \mathrm{~cm}$, frequency $5.4 \mathrm{GHz}$. Compares of the results are shown below, and the experimental data could find in the reference book wrote by Yingzheng Ruan[17].

BLV: total width $2.96 \mathrm{~m}$, total length $6.5 \mathrm{~m}$, frequency $9.0 \mathrm{GHz}$.

As shown in figure 9, the result of cylinder of the self-developed program fits the experimental data quite good with the same fluctuating characteristics in detail and the same trend on the whole, but the value is generally small, it could be due to the lack of edge diffraction. For the vehicle designed in this paper, result presented in figure 10 reflects the symmetrical features of the vehicle and is very close to the result of commercial software. In conclusion, the RCS estimation procedure could predict the electromagnetic scattering exactly, and possess high practical value.

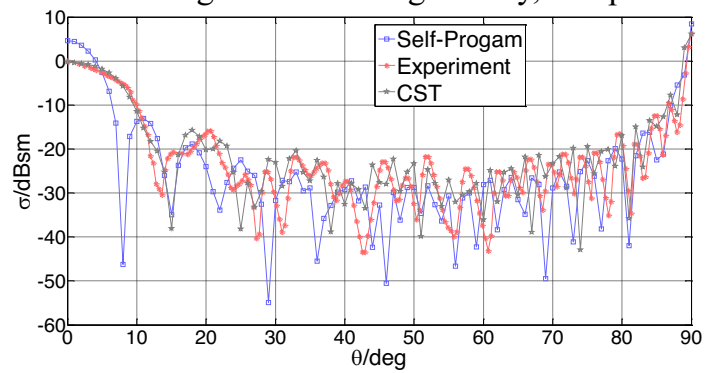

Figure 9. Monostatic RCS compare of the cylinder with different data

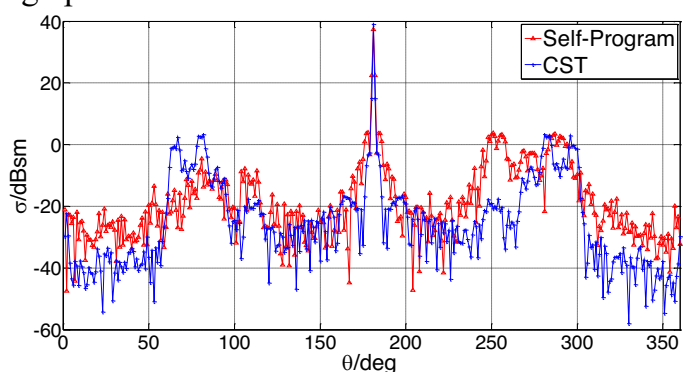

Figure 10. Monostatic RCS compare of the BLV with Self-Program and CST data

\section{Integrated design of aerodynamic and stealth}

The technology of 3D geometric modeling, rapid grid generation, read and transformation of grid data, rapid RCS calculation, and rapid aerodynamic calculation is involved in the integrated design. In this paper, integrated platform of the hypersonic BLV could link the output from the aircraft shape control parameters with the aerodynamic characteristics and RCS characteristic data automatically.

As shown in Figure 11, when the aircraft shape control parameters are changed, the CATIA module updates the model and the display automatically, and then mesh module according to grid needs of aerodynamic and RCS calculation generates two sets of grid files, aerodynamic branch read grid files for aerodynamic and calculate for the aerodynamic coefficient, and the RCS branch reads the RCS grid to obtain the average RCS in the forward direction of the vehicle. 


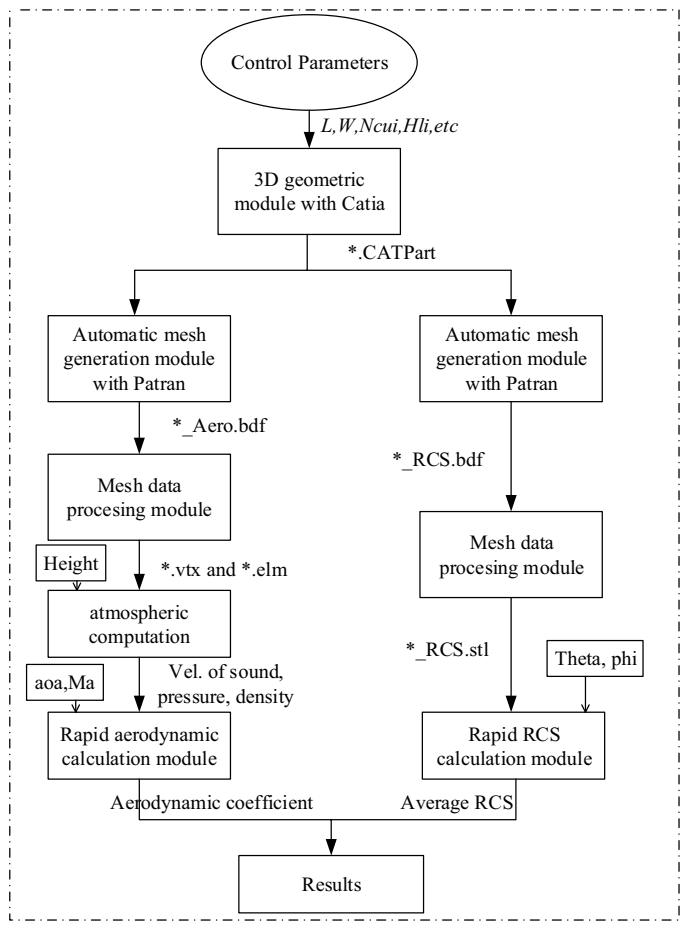

Figure 11. The work flow of the aerodynamic/stealth integrated platform

\section{Sensitivity analysis of control parameters}

Sensitivity analysis is a branch of mathematical statistics, which is one of the most important statistical methods in optimization nowadays. With appropriate sensitivity analysis before optimization, twice as much can be accomplished with half the expenditure.

The Orthogonal Arrays (OA) is an efficient, rapid and economical method for fractional factorial analysis. It is a set of combination of multiple factors at multiple levels which are balanced to ensure that all levels of all factors are considered equally. Dr. Genichi Taguchi has designed a group of matrix filled with OA of 2 or 3 levels, with which the experiments could be applied more scientific.

As there are 23 parameters of the blended-wing-body lifting vehicle, but only 17 of them is alterable, an experiment of 17 factors at 3 levels (the low level is the lower limit, the high level is the upper bound, and the middle level is the average of bounds. ) is carried out for sensitivity analysis on RCS and aerodynamic performance. The results are presented below:
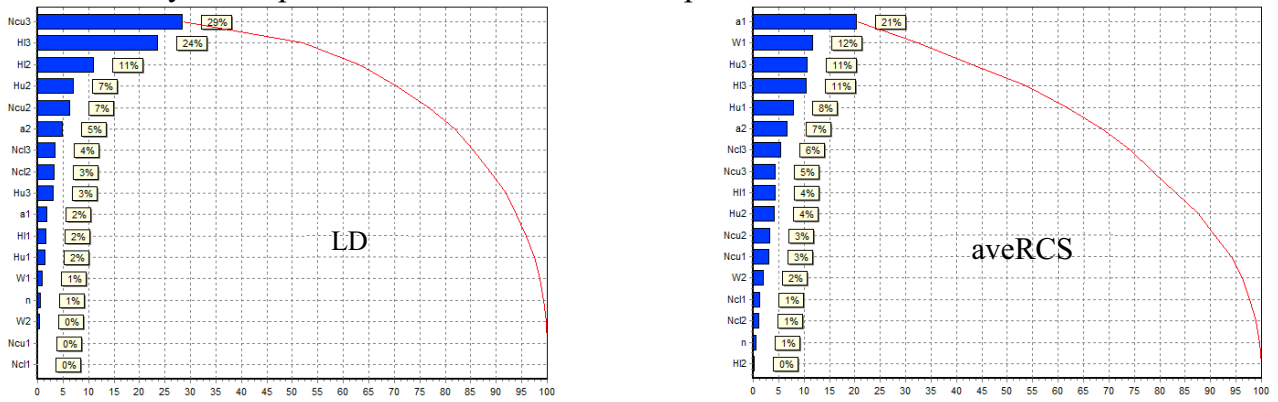

Figure 12. The results of sensitivity analysis on LD and average RCS.

The influence of control parameters on LD or average RCS is expressed as percentage shown in figure 12 , assume that value greater than $7 \%$ indicates a significant effect, thus the variables have a 
remarkable impact on LD are $N_{c u 3}, H_{l 3}$, and $H_{l 2}$, and on RCS are $a_{1}, w_{1}, H_{u 3}$, and $H_{l 3}$. It's easy to figure out the reason why $N_{c u 3}$ and $H_{l 3}$ is notable to LD, both $N_{c u 3}$ and $H_{l 3}$ are the control parameters of the biggest cross section, which means the greatest impact on the shape in the group of $N_{c u i}$ and $H_{l i}$.

The effects of shape control parameters on RCS is vital, as it is difficult to master the influence of shape on RCS. Both LD and RCS are impacted definitely by $H_{l 3}$, which means $H_{l 3}$ should be carefully considered in further design. According to the result of sensitivity analysis of control parameters, there is no great conflict between the aerodynamic and stealth, which shows that the overall performance of the vehicle could be better after optimization.

\section{Conclusion}

In this paper, a blended-wing-body lifting vehicle is designed by mixing three kinds of curve control method, meanwhile a platform for integrated design and analysis is built. With the help of the platform, parameters which are prominent in the aerodynamic and radar reflection characteristics are mastered by sensitivity analysis, thus it becomes clear that how to design a vehicle with particular aerodynamic and radar needs. The further work would be concentrated on raising and solving the MOP of aerodynamic and stealth, and improving the precision of each module to make the results more reliable.

\section{Acknowledgments}

This work is supported by Aeronautical Science Foundation of China (Grant No. 2016ZA880003).

\section{References}

1. T. Smith, K. Bowcutt, J. Selmon, et al. HIFiRE 4: A Low-Cost Aerodynamics, Stability, and Control Hypersonic Flight Experiment [C]. 17th AIAA International Space Planes and Hypersonic Systems and Technologies Conference. (San Francisco, California. 2011).

2. L. Zhang. Rapid aerodynamic shape optimization design of aircraft based on surrogate model [D]. Changsha, Hunan, P.R. China: National University of Defence Technology (2015).

3. J. Hu, R.H. Wang, W.J. Wang, et al. Multidisciplinary Optimization of Transport Wing Aerodynamic/Structural Integrated Design [J]. J. Nanjing Univ. Aero. \& Astro. 44, 458-463 (2012).

4. X. Chen, L. Liu, Z.J. Yue. A Reduced Order Modeling for Aerothermodynamic of Hypercsonic Vehicles Based on Surrogate Method [J]. Tran. Beijing Ins. Tech. 36, 340-348 (2016).

5. Q. Gao, J.B. Zhao, Q. Li. Study on Lateral-Directional Stability of HTV-2 Like Configuration [J]. J. Astro. 36, 657-662 (2014).

6. X.Y. Chu. Integrated Design Method of Cruise Missile Including Configuration, Aerodynamics and Radar Stealth [D]. Nanjing: Nanjing University of Aeronautics and Astronautics. (2004).

7. Z.H. Liu, P.L. Huang, X. Gao, et al. Multi-frequency RCS Reduction Characteristics of Shape Stealth with MLFMA with Improved MMN [J]. Chin. J. Aero. 23, 327-333 (2010).

8. W. Di, L. Teng, L.Y. Lin et al. Aero-Structure-Stealth Coupled Optimization for High Aspect Ratio Wing Using Adaptive Metamodeling Method [C]. 15th AIAA/ISSMO Multidisciplinary Analysis and Optimization Conference. (Atlanta, GA. 2014).

9. J.X. Su, Y. Lu, Z.Y. Zheng et al. Fast analysis and optimal design of metasurface for wideband monostatic and multi-static radar stealth [J]. J. Appl. Phys. 120, (2016).

10. X.W. Jiang, Q.J. Zhao, G.G. Zhao et al. Integrated optimization analyses of aerodynamic/stealth characteristics of helicopter rotor based on surrogate model [J]. Chin. J. Aero. 28, 737-748 (2015). 
11. J. Hu, T. Liu, P. Zhang et al. Aerodynamics/Structure/Invisibility Integrative Design and Multidisciplinary Design Optimization of Loitering Weapon [J]. Aero Weap. 3, 9-13 (2016).

12. Z.H. Jiao, F. Deng, H. Liu et al. Aerodynamic and Stealthy Optimization Design Method of Hypersonic Vehicle [J]. J. Astro. 39, 1031-1040 (2016).

13. K. Brenda. A universal parametric geometry representation method - "CST" [C]. 45th AIAA Aerospace Sciences Meeting and Exhibit. (Reno, Nevada. 2007).

14. K. Brenda. Universal parametric geometry representation method [J]. J. Airc. 45, 142-158 (2008).

15. K. Brenda, B. John. "Fundamental" parametric geometry representations for aircraft component shapes [C]. 11th AIAA/ISSMO MAOC. (Portsmouth, Virginia. 2006).

16. C. Filippos. Development of code for a physical optics radar cross section prediction and analysis application [D]. California: Naval Postgraduate School. (2004).

17. Y.Z Ruan. Radar Cross Section and Stealth Technology [M]. Beijing:National Defence Industry Press. (1998). 